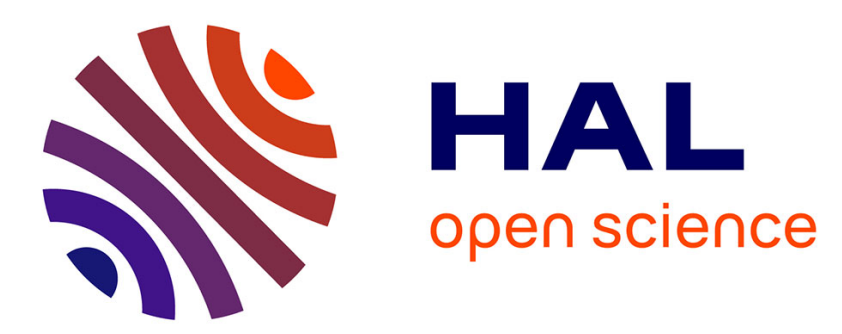

\title{
A grid-enabled algorithm yields Figure-Eight molecular knot
}

Fotis Evangelos Psomopoulos, Pericles A Mitkas, Christos S Krinas, Ioannis Nikolaos Demetropoulos

\section{> To cite this version:}

Fotis Evangelos Psomopoulos, Pericles A Mitkas, Christos S Krinas, Ioannis Nikolaos Demetropoulos. A grid-enabled algorithm yields Figure-Eight molecular knot. Molecular Simulation, 2009, 35 (09), pp.725-736. 10.1080/08927020902833103 . hal-00515082

\section{HAL Id: hal-00515082 https://hal.science/hal-00515082}

Submitted on 4 Sep 2010

HAL is a multi-disciplinary open access archive for the deposit and dissemination of scientific research documents, whether they are published or not. The documents may come from teaching and research institutions in France or abroad, or from public or private research centers.
L'archive ouverte pluridisciplinaire $\mathbf{H A L}$, est destinée au dépôt et à la diffusion de documents scientifiques de niveau recherche, publiés ou non, émanant des établissements d'enseignement et de recherche français ou étrangers, des laboratoires publics ou privés. 


\section{Molecular Simulation}

Journal of

Experimental Nanoscience

$\because$ Taylor \& Francis

Taglor S Francis Group

\section{A grid-enabled algorithm yields Figure-Eight molecular knot}

\begin{tabular}{|r|l|}
\hline Journal: & Molecular Simulation/Journal of Experimental Nanoscience \\
\hline Manuscript ID: & GMOS-2008-0236.R1 \\
\hline Journal: & Molecular Simulation \\
\hline Date Submitted by the & 14 -Feb-2009 \\
\hline Author: & $\begin{array}{r}\text { Mitkas, Pericles; Aristotle University of Thessaloniki, Electrical and } \\
\text { Computer Engineering } \\
\text { Krinas, Christos; Democritus University of Thrace, Molecular Biology } \\
\text { and Genetics } \\
\text { Demetropoulos, Ioannis; University of Western Macedonia, } \\
\text { Department of Engineering Informatics and Telecommunications }\end{array}$ \\
\hline Keywords: & $\begin{array}{l}\text { Knot Theory, Stereochemistry, Grid Computing, Data } \\
\text { Decomposition, Figure-Eight Molecular Knot }\end{array}$ \\
\hline \hline
\end{tabular}

\section{SCHOLARONE Manuscripts}




\begin{abstract}
The recently proposed General Molecular Knotting algorithm and its associated package, MolKnot, introduce programming into certain sections of stereochemistry. This work reports the g-MolKnot procedure that was deployed over the Grid infrastructure; it applies a divide-and-conquer approach to the problem by splitting the initial search space into multiple independent processes and, combining the results at the end, yields significant improvements with regards to the overall efficiency. The algorithm successfully detected the smallest ever-reported alkane configured to an open-knotted shape with four crossings.
\end{abstract}

Key words: Knot Theory, Stereochemistry, Grid Computing, Data Decomposition, Figure-Eight Molecular Knot

\section{Introduction}

A particular spatial entanglement of a molecule is a mechanically-interlocked molecular architecture that is analogous to a macroscopic knot (Figure 1). Molecular knots are also referred to by some chemists as "knotanes". The term knotane was coined by Fritz Vögtle et al [1] by analogy with rotaxane and catenane. Knotanes are increasingly found in nature (knotted proteins [2] [3] and DNA [4]) while synthesized molecular knots [5] [6] along

* Corresponding author; Tel: +30 231099 6349, Fax: +30 2310996398

** Corresponding author; Tel: +30 302461056540

Email addresses: fpsom@issel.ee.auth.gr (Fotis E. Psomopoulos), mitkas@eng.auth.gr (Pericles.A.Mitkas),me00599@cc.uoi.gr (Christos S.Krinas), idimitr@uowm.gr (Ioannis N. Demetropoulos)

URLs: http://issel.ee.auth.gr/en/fpsom (Fotis E. Psomopoulos), http://issel.ee.auth.gr/en/mitkas (Pericles A. Mitkas). with catenanes and rotaxanes have been proposed as parts of potential molecular machines [7] [8] [9] or scaffolds [10] for drug carriers. Nowadays molecular modeling, such as computational materials and drug discovery, are among the largest consumers of CPU power (probably excluding military applications second only to weather prediction) [11].

Existing molecular knot construction methods [12] [13] [14] [15] [16] are considered inadequate for short molecular chains. Recently we proposed a de novo computer-aided systematic production of small molecular knots with three or more crossings and unlinked ends [17]. The generalized molecular knotting algorithm (GMK) was implemented in the public domain code MolKnot [18]. The GMK algorithm narrows the knotted conformer search to a sub domain of the total
Deleted: 2

Deleted: 3 
conformational space (TCS) which is most likely to contain knotted conformers according to the topological theory of open polygonal knots [19] [20]. The algorithm itself is amenable to domain decomposition, so the program has been modified in order to run in a computational grid.

Most of the modeling in physics and chemistry is of the type of Quantitative Structure-Activity Relationship (QSAR) [21], which describes how a chemical structure is quantitatively correlated with a well defined property, such as biological activity, chemical reactivity or physical property. The general mathematical form of the QSAR is as follows (Eq. 1):

Activity $=f$ (physicochemical properties and / or structural properties)

Length, Number of linear Segments) [17]. Furthermore it has been shown [27] that knottiness induces bond elongation and valence angle distortion so a possible QSAR is reactivity $=$ f(knottiness, bond elongation, valence angle distortion).

Since the problem of finding the property's value on the sub domains (different molecules or conformers) are independent to each other, the QSAR construct is ideally suited as a GRID computing application.

The structure of this article is as follows. Section 2 describes the implementation of the gridification of the Mol-Knot program, Section 3 presents a case study from the homologous hydrocarbon series $\mathrm{C}_{n} \mathrm{H}_{2 n+2}\left(\mathrm{C}_{36} \mathrm{H}_{74}\right)$ with emphasis to $4_{1}$ knots. Finally the main findings of the work are summarized in Section 4.

solubility, lipophilicity, (expressed as an energy function minimum), dipole moment etc, is distinct for each property. For instance: (A) a QSAR connects the dynamic viscosity $(\eta)$ of dilute solutions of the triglycerides to the viscosity of the solvent (no), the triglycerides' concentration (C) and the structural characteristics, such as the length of the carbon chains (CN) and the number of double bonds (DB) via the simple empirical equation $\ln (\eta)=k_{0}$ $+\mathrm{k}_{1} \ln \left(\eta_{0}\right)+\mathrm{k}_{2} \mathrm{CN}+\mathrm{k}_{3} \mathrm{DB}+\mathrm{k}_{4} \mathrm{C}[22]$. (B) $\mathrm{a}$ QSAR on enzymic activity could be an inequality constraint. Root Mean Square (RMS) distances between the heavy atoms of the side chains of certain catalytic triad residues of an enzyme should be less than $2 \AA$ [23] so an enzymomimetic compound [24] could fulfill the spatiotemporal criterion [25]. In maths the knottiness of a polygonal line is related to certain relationships between its linear segments [19] [20]. The molecular knottiness is the conformers' subspace (Total Knotted Conformer Space - TKCS - of a compound). TKCS is a function of the macromolecule's length and the number of its linear segments. Hence the related topological indicator is knottiness $<f($ Macromolecules'

\section{Computational Methods}

\subsection{The GMK algorithm}

An interesting aspect of linear alkane molecules is that they have the ability to fold, thus creating knots in 3-dimensional space. The molecule chain can be modeled as a polygonal line, allowing for an algorithmic approach to the problem of detecting the specific segment that can produce a knot. The Generalized Molecular Knot algorithm is a four-step computational approach that targets open knot formations. This original procedure is as follows:

(i) Conformer Generation Step: Molecular conformations with inherent knot topology are created by applying the theory of open polygonal knots at dihedral space,

(ii) Math Filter Step:

Each structure is evaluated for knottiness by calculating the Alexander polynomial $\Delta(\mathrm{t})[26]$ [17] [27],

Formatted: Font: Italic
Formatted: Font: $11 \mathrm{pt}$
Deleted: 4
Deleted: 15
Formatted: Font: Italic

Deleted: 16

Deleted:

Formatted: Subscript

\begin{tabular}{|l|} 
Formatted: Subscript \\
\hline Formatted: Subscript \\
\hline Formatted: Subscript \\
Formatted: Subscript \\
Formatted: Subscript \\
Formatted: Subscript
\end{tabular}




\section{Page 3 of 24}

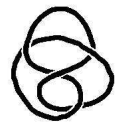

[a]

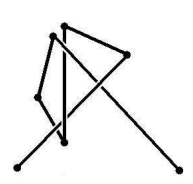

[d]

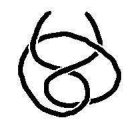

[b]

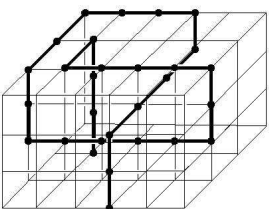

[e]

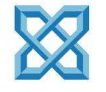

[c]

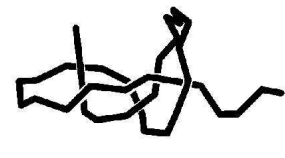

[f]

Fig. 1. Figure-Eight knots in various settings. [a] a rope shaped as 41 Knot (figure-eight knot), [b] a rope with open ends, [c] a 41 knot using two closed hexagons (also known as King Solomon's knot), [d] a polygonal chain with 7 vertices in open knot 41 formation, [e] open figure-eight knot constructed with 29 vertices (which comprise 11 linear segments with lengths as follows: 2, 3, 1, 3, 2, 2, 5, 2, 4, 1 and 3), and [f] an alkane as an open figure-eight knot.

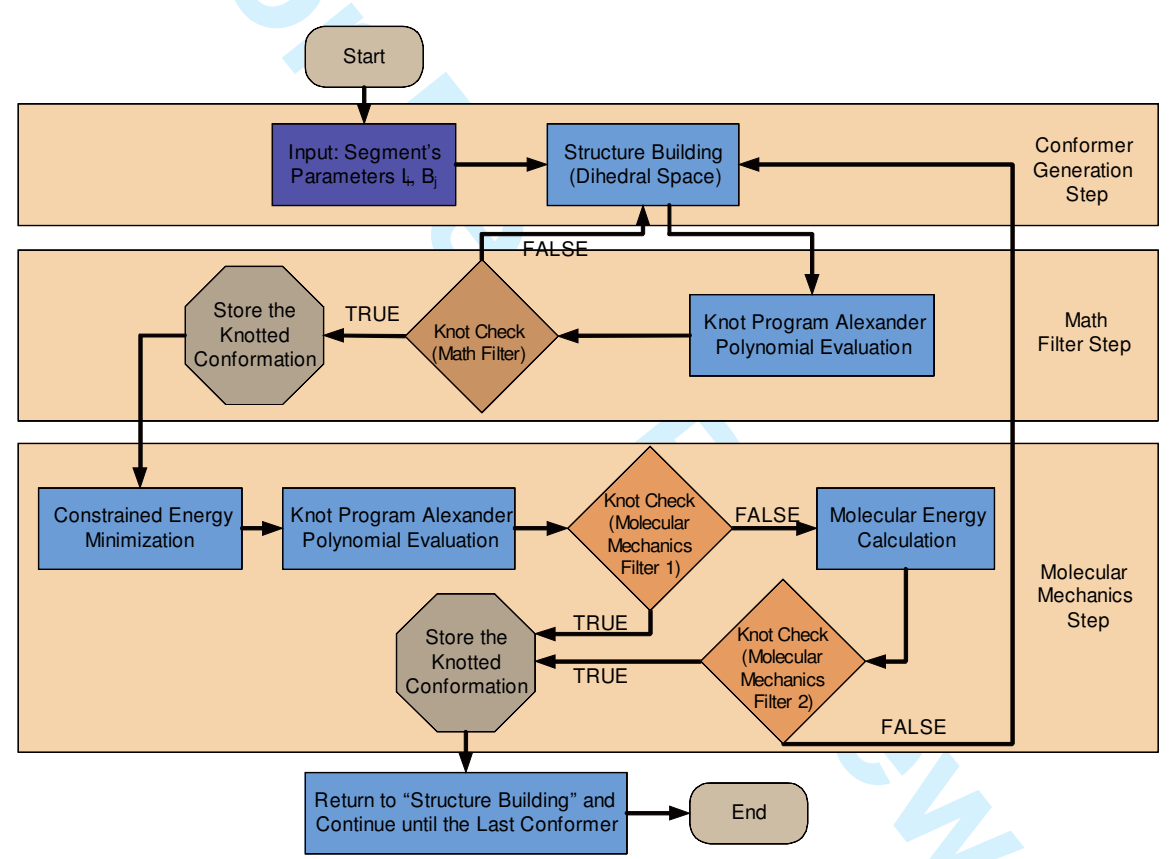

Fig. 2. Logical diagram of the GMK algorithm. The conformers are saved as open polygonal knotted graphs and as molecular mechanics' optimized structures. This figure clearly depicts the three initial steps as described in Section 2.1. Molecular Mechanics Filter 1 decides based on the Alexander Polynomial evaluation for a geometry optimized using the Molecular Mechanics description. Molecular Mechanics Filter 2 decides based on the energy value assigned to the conformer under examination. A high energy value compared to the energy of the extended $\mathrm{C}_{36} \mathrm{H}_{\mathbf{2} 2}$ hydrocarbon molecule implies that the conformer is entangled. The energy criterion is especially discriminating for tight knots.

(iii) Molecular Mechanics Step:

The confirmed knots are subjected to constraint geometry optimization using classical molecular mechanics (MM) force field equations, and are then reevaluated for knottiness, and

\begin{tabular}{|l|}
\hline Formatted: Subscript \\
\hline Formatted: Subscript \\
\hline
\end{tabular}




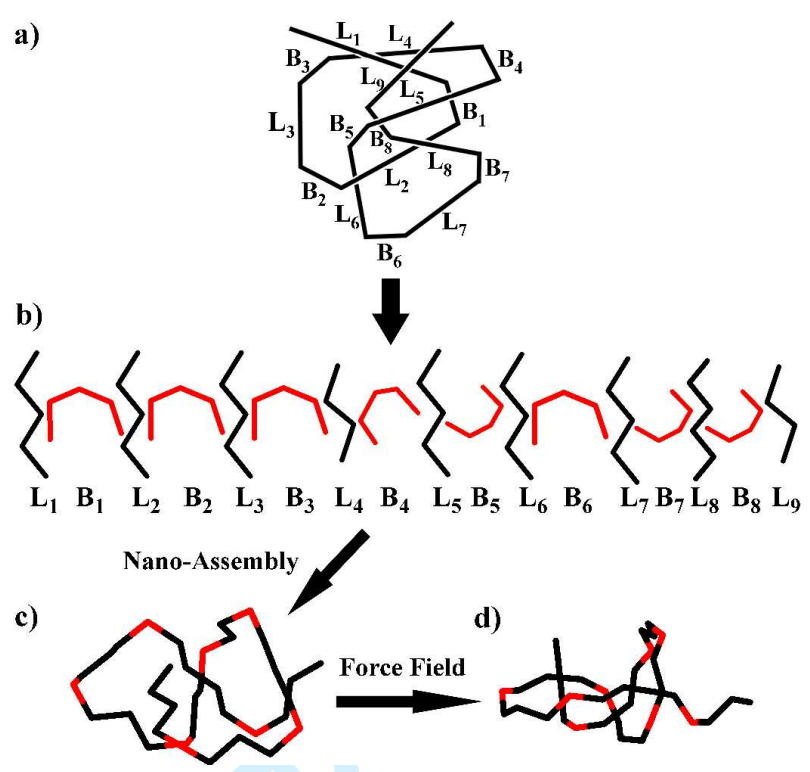

Fig. 3. A graphical presentation of the GMK algorithm as applied to $\mathrm{C}_{36} \mathrm{H}_{74}$ molecule for $\mathrm{k}=9$. (a) The model with 9 straight segments $\mathrm{L}_{\mathrm{i}}$ and 8 turning points $\mathrm{B}_{\mathrm{i}}$, (b) Molecular segments used for the assembly of the initial molecular geometry $\left(\mathrm{L}_{\mathrm{i}}\right.$, black color), $\left(\mathrm{B}_{\mathrm{i}}\right.$, red color, (c) The initial building sequence $\Phi_{1}, \Phi_{2}, . ., \Phi_{33}$, where $\Phi_{1}, \Phi_{2}, . ., \Phi_{33}$ are CCCC torsion angles in the carbon backbone of the $\mathrm{C}_{36} \mathrm{H}_{74}$ molecule (d) Tube representation of the outcome of the geometry optimization procedure of the $\mathrm{C}_{36} \mathrm{H}_{74}$ hydrocarbon; the sequence of CCCC backbone torsion angles. Appendix A contains the specifics of steps

| $\mathrm{b}],[\mathrm{c}]$ and $[\mathrm{d}]$ steps that may allow the interested reader to reproduce the transformations. The conformer maintained its entanglement at $\mathrm{B} 3 \mathrm{LYP} / 631 \mathrm{G} * *$ optimization level. For interpretation of the references to color in this figure legend, the reader is referred to the web version of this article.

(iv) Quantum Physics Step:

The resultant structures are subjected to unconstrained geometry optimization at a semi-empirical quantum level (AM1).

The flow chart of the GMK algorithm showing the main conceptual steps in the process, can be seen in Figure 2. This chart does not include the quantum step since that only serves as a shape optimizer [1]_ [27] - knottiness is generally preserved. Moreover, the first three steps of the algorithm are the most computationally expensive. Hereby we define lengths at the dihedral space of linear molecules. The polyethylene molecule
$\mathrm{C}_{\mathrm{n}} \mathrm{H}_{2 \mathrm{n}+2}$ is modeled as a finite number of straight line segments that are linked together with suitable turns. Line segments are represented as contiguous $180^{\circ}$ CCCC torsion angles, and the turns are $-\left(\mathrm{CH}_{2}\right)_{5}-$ fragments with two contiguous torsion angles (Figures $3[\mathrm{a}]$ and $3[\mathrm{~b}]$ ). In practice, this means that the angle defined by three consecutive atoms $\mathrm{C}_{\mathrm{i}}, \mathrm{C}_{\mathrm{i}+1}, \mathrm{C}_{\mathrm{i}+2}$ is rigid (i.e. is not permitted to change more than $\pm 5^{\circ}$ ), whereas the dihedral angle defined-by four-consecutiveatoms $\mathrm{C}_{i}, \mathrm{C}_{\mathrm{i}+1}, \mathrm{C}_{\mathrm{i}+2}, \mathrm{C}_{\mathrm{i}+3}$ is flexible, by allowing the rotation along the axis defined by $\mathrm{C}_{\mathrm{i}+1}$ and $\mathrm{C}_{\mathrm{i}+2}$. This is the definition of "dibedral angle" between four points.

\section{Deleted:}

Deleted: 2

Deleted: 18 
1

2

3

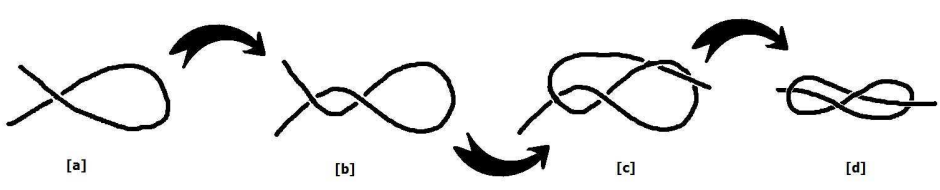

Fig. 4. Figure-of-Eight or Flemish Knot. The rhyme to forming the knot is "Twist it once, twist it twice; pass it through and make it nice" [28].

Without getting into detail, each generated molecular conformation must pass through two consecutive filters. First a mathematical filter rejects any formation that is not knotted (i.e. the formation must adhere to a knot topology, as shown in Figure 3[c]). Second a molecular mechanics filter allows only the mathematical knots which are physically accepted by means of a multi-spring model (i.e. the molecule is described as a multi-spring device). The algorithm has been shown to be quite efficient for short chain lengths, where all other methods fail [27]. The buildup of a $4_{1}$ knot at molecular level is illustrated in Figure 3, while the sequence of twists that lead to a FigureEight knot, realized in a rope, is presented in Fig. 4.

The fraction of the knotted conformers (total knotted conformational space - TKCS) over all permitted possibilities for the total conformational space (TCS) is a measure of the Delbrücks [29] using Eq. (2), where $n c$ is the number of carbons in the molecular chain, and $k$ is the number of segments in the polygonal line model.

As an example, the TCS size of $\mathrm{C}_{36} \mathrm{H}_{74}$ is enormous $\left(1.5 \times 10^{17}\right)$ while TKCS is 7 orders of magnitude smaller $\left(5.6 \times 10^{10}\right)$. By removing segment lengths that are less likely to produce tight knots, the TKCS can be further reduced to $5.5 \times 10^{8}$. In [27] the TCS size of $\mathrm{C}_{30} \mathrm{H}_{62}$ is stated to knotting probability. The TKCS can be evaluated

It is obvious that the process can be very time consuming, as the size of the molecule in question increases. On the other hand, the sequential approach to the problem may not be optimal, due to the fact that each generated conformation could potentially go through both phases, although evaluations of molecular conformations are independent. In fact, the conformations can be regarded as the "data" of the process, while the rest of the program can be viewed as a single function, thus characterizing the GMK algorithm as a data decomposition process. Therefore, as an embarrassingly parallel process, GMK can only gain through deployment over a Grid environment.

\subsection{Grid and MolKnot}

Although the term "Grid" has been conflated, at least in popular perception, to embrace anything from advanced networking to artificial

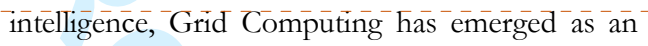
important new field in computer science. Foster [30] gives the following definition of the Grid:

A computational grid is a hardware and software infrastructure that provides dependable, consistent, pervasive, and inexpensive access to high-end computational capabilities.

In practice, a Grid environment can be perceived as a virtual computing architecture that provides -the - ability - -to - perform - higher - throughput-

$$
\operatorname{TKCS}(n c, k)=\sum_{k=5}^{\lfloor((n c-1) / 3\rfloor}\left(\frac{(n c-2 k-2) !}{(k-1) !(n c-3 k-1) !} \times 6^{k-1}\right)
$$

be $9.9 \times 10^{14}$ while TKCS is reported to be 7 orders of magnitude smaller $(16,656,192)$.

computing by taking advantage of many computers geographically dispersed and connected by a network. Currently, there exist
Deleted: 20

Deleted: 21
Deleted: 18

Deleted: 
several major Grid middleware providers, such as EGEE [31], UNICORE [32], Globus [33] (Europe, USA) and Vega [34] (CNGrid). However, the differences in software are not reflected on the actual hardware infrastructure. In fact, there is an ongoing effort of unifying the Grid infrastructures worldwide. The first step toward this direction was the interoperability of the European and USA middlewares, namely EGEE, UNICORE and Globus. There are several projects that aim to integrate the European infrastructure with others, such as EUChinaGrid (which provides specific support actions for the interoperability of EGEE and CNGrid), and the EUIndiaGrid project (which will make available a common infrastructure to the European and Indian scientific community). Due to this interoperability initiative, the g-MolKnot application is expected to run without any problems in other Grid infrastructures, but this has yet to be tested.

g-MolKnot was developed based on the MolKnot algorithm, aiming to create an implementation that would take full advantage of a Grid environment. Due to the embarrassingly parallel nature of the algorithm (as will be discussed later), the implementation is not based on the MPI Library, thus enhancing the portability of the application. The design paradigm falls into the parameter sweep category; the conformational range is divided into a number of disjoint sub-ranges and each sub-space is assigned to a different process, which performs the MolKnot procedure through all steps. Finally, the results are merged together, making the whole process transparent to the enduser. Although the divide-and-conquer approach has been proved efficient several times in parameter sweeping problems [35], it stands to reason that a decomposition of the MolKnot procedure into multiple independent processes will allow for a significant gain in execution time.

\subsection{GMK Complexity analysis}

As shown in the previous section, the GMK algorithm can be decomposed into three independent steps. An initial approximation to the overall complexity of the algorithm is the following: Given a conformation space $V$ of a molecule with $n c$ number of carbons, the number of generated molecular formations will be $N=$ $|V|$ ( $N$ takes the value of TKCS). In the worst case scenario, where all generated formations are indeed actual knots, the sequential approach will have to make $N$ iterations through the first three phases. Without loss of generality, the complexity of the first phase can be assumed to be equal to $\mathrm{O}(N \times n c)$, the complexity of the second phase equal toO $\left(N \times n c^{2}\right)$, and the complexity of the third phase equal to $\mathrm{O}\left(N \times n c^{3}\right)$. Hence, the sequential complexity would be approximately equal to $\mathrm{O}\left(\sum_{k=1}^{3} N \times n c^{k}\right)$. Using the $\mathrm{g}$-MolKnot approach, the same space $V$ will be decomposed to $p$ disjoint sub-spaces, each of which will be processed independently by one of the $p$ requested CPU units. Thus the complexity becomes approximately equal to $\mathrm{O}\left(\sum_{k=1}^{3} \frac{N \times n c^{k}}{p}\right)$. In practice however, the number of formations that successfully pass through each filter decrease exponentially with each phase. That is, if $V_{i}, i=1$, 2,3 , is the conformation space at the end of phase

$i$, with $V \supseteq V_{1} \supseteq V_{2} \supseteq V_{3}$, then $|V|>>\left|V_{1}\right|$

$>>\left|V_{2}\right|>>\left|V_{3}\right|$, thus leading to a significant reduction in complexity, as shown in the experiments in the next section.

Eq. (3) shows the complexity of the first three steps of GMK for a single conformation as a function of $n c$.

\begin{tabular}{|l|}
\hline Deleted: 22 \\
\hline Deleted: 23 \\
\hline Deleted: 24 \\
Deleted: 25 \\
\hline
\end{tabular}




$$
\begin{aligned}
O(n c)= & \underbrace{z \times O(n c)}_{\text {Molecule Generation }}+ \\
& \underbrace{x \times O\left(n c^{2.376}\right)}_{\text {Math Filter }}+ \\
& \underbrace{\left[y_{1} \times O\left(n c^{2}\right)+y_{2} \times O\left(n c^{2}\right)+y_{3} \times O\left(n c^{3}\right)\right]}_{\text {Molecular Mechanics Filter }}
\end{aligned}
$$

Experimentally, $z, x, y 1, y 2$ and $y 3$ take the following values: $\mathrm{z}=\mathrm{x}=1, \mathrm{y} 1 \approx 900, \mathrm{y} 2 \approx 800$ and $y 3 \approx 1200$. The complexity of the second term is given according to Coppersmith and Winograd [36].

In a real-world example, the number of conformations $N$ is a significant factor in the overall complexity of the problem, transforming Eq. (3) into the following:

$$
\begin{aligned}
O(n c, N)=\ln ^{\frac{2}{3}}(N) \times[\underbrace{z \times O(n c)}_{\text {Molecule Generation }}+ & \underbrace{x \times O\left(n c^{2.376}\right)}_{\text {Math Filter }}+ \\
& \underbrace{\left[y_{1} \times O\left(n c^{2}\right)+y_{2} \times O\left(n c^{2}\right)+y_{3} \times O\left(n c^{3}\right)\right]}_{\text {Molecular Mechanics Filter }}] \\
O(n c, N, a, b)=\ln ^{\frac{2}{3}}( & N) \times[z \times O(n c)+ \\
& x \times O\left(n c^{2.376}\right)+ \\
& {\left[\frac{a}{N} \times\left(1-\frac{b}{a}\right) \times\left(y_{1} \times O\left(n c^{2}\right)+y_{2} \times O\left(n c^{2}\right)+y_{3} \times O\left(n c^{3}\right)\right)\right]+} \\
& {\left.\left[\frac{a}{N} \times \frac{b}{a} \times\left(y_{1}^{\prime} \times O\left(n c^{2}\right)+y_{2}^{\prime} \times O\left(n c^{2}\right)+y_{3}^{\prime} \times O\left(n c^{3}\right)\right)\right]\right] }
\end{aligned}
$$

Finally, a thorough complexity analysis will require a closer look at the design of the molecular mechanics filter, which comprises three serial iterative procedures. In the worst case scenario, a single molecular conformation that would fail the filter will have to exhaust all available iterations, as opposed to a knotted conformation, which will pass through the filter using a small number of iterations. rejected by the filter, which applies to the_majority of the conformers, and it is usually captured by the first steps. The second part expresses the case when a conformation is accepted by the filter, which means that has successfully passed all three steps. However, in this case each step is passed relatively quickly, and this is reflected in the values of $y_{1}{ }^{\prime}, y_{2}{ }^{\prime}$ and $y_{3}{ }^{\prime}$. Overall, it can be seen that the worst case
Deleted: 27 
is $\mathrm{O}(n c, N)=\ln ^{\frac{2}{3}}(N) \times O\left(n c^{3}\right)$. The coefficients in the analytical form of the complexity suggest that the actual complexity is lower and closer to 2 than 3. Moreover, the complexity can be further reduced by optimizing the steps that constitute the molecular mechanics filter, so as to reduce the coefficient of the $\mathrm{O}\left(n c^{3}\right)$. By splitting the data (i.e. the conformations) into multiple processes $p$, the new complexity of the parallel procedure will be:

$\mathrm{O}(n c, N, p)=\frac{\ln ^{\frac{2}{3}}(N) \times O\left(n c^{3}\right)}{p}$

which clearly indicates the validity of porting the GMK algorithm to a Grid environment. This analysis will be an important feedback for a better tuning of the program, by increasing the ratio of $\mathrm{y}_{1}, \mathrm{y}_{1}{ }^{\prime}, \mathrm{y}_{2}, \mathrm{y}_{2}{ }^{\prime}$ against $\mathrm{y}_{3}, \mathrm{y}_{3}{ }^{\prime}$. In this way future runs will perform better.

\section{Results and Discussion}

\subsection{Computational Experiment Setup}

For the experiment, a subspace of Eq. (2) will be used (SubKCS $(n c, k))$, by allowing only a single value of k. Moreover, instead of all 6 available values of bends, only 4 where allowed. Therefore, Eq. (2) is transformed as follows:

$$
\operatorname{SubKCS}(n c, k)=\left(\frac{(n c-2 k-2) !}{(k-1) !(n c-3 k-1) !} \times 4^{k-1}\right)
$$

The experiment design focused on the $\mathrm{C}_{36} \mathrm{H}_{74}$ molecule with $n c=36$ and the polygonal line comprising of $k=9$ segments (as discussed in Section 2). These parameters lead to a total conformational space of approximately $3.5 \times 10^{9}$. However, a further reduction of the search space was deemed necessary, so that the acceptable segment lengths of the polygonal line would be limited to $1,2,3$ and 4 . The size of this new subspace is $553,844,736$ (order of $0.5 \times 10^{9}$ ), which is an order of magnitude larger than any other in literature so far [27] [17].This means that the corresponding computational power required for the experiment is also high [20]. For this reason, the "gridification" of the process is justified.

The experiment allows for a fairly complete search of the conformational space of the molecular chain with 36 carbonatoms (dihedral length 33). A longer molecular chain provides the ability to search for knotted conformations of greater complexity than those studied in existing literature. However, even this length is restrictive for higher complexities (such as 5 crossings or more). Despite this fact, this paper focuses to search for knots with 4 crossings, which was not possible with previously studied lengths. In this section, the results of the $\mathrm{g}$-MolKnot application are presented, in order to evaluate its effectiveness and efficiency. There is also a discussion on the correlation of the conformational space TKCS searched with the number of produced knotted conformers at each filter and their complexity (i.e. knots with 3 or 4 crossings).

\subsection{Optimization of run-time parameters for Grid} execution

Table 1

The number of produced knotted conformers after each filter for the different subspaces. Notice the correlation between the output of the mathematics and the molecular mechanics filters.

\begin{tabular}{ccc}
\hline \hline \# of conformations & \# Pass Math Filters & \# Pass MM Filter \\
\hline 1,000 & 23 & 5 \\
5,000 & 78 & 7 \\
10,000 & 125 & 11 \\
50,000 & 403 & 26 \\
100,000 & 425 & 29 \\
\hline
\end{tabular}

The analysis of the Grid parameters is performed in order to define the optimal parameters for the final run. On one hand, the major issue in the gridification of the process is to define the number of subspaces which will be assigned to the independent processes on the Grid with regards to time efficiency. On the other hand, the effect of the _heterogeneity of _ Grid _ resources _ on - the application should also be studied.
Deleted: 15

Deleted:

Deleted:
Deleted:
Deleted: 18
Deleted: 2


Fig. 5. The first diagram shows the average performance of the algorithm in 1, 10, 20 and 40 CPUs. In all other diagrams, the red line is the average performance in each case, with the green-dotted lines above and below showing the worst and best processing time respectively. In a homogeneous experiment the dotted lines converge to the average line.

The first step in the optimization process is to verify in practice that the domain decomposition, as presented in Section 2, is justified as an optimization process for the specific problem. To this aim, a smaller dataset which comprised a 27 carbon atoms alkane and only part of its conformational space was used. Specifically, the molecule was searched in 5 different subspaces, containing $1,000,5,000,10,000,50,000$ and 100,000 conformations, Since the time consumption, as defined in Section 2.3, is related to the production of knotted conformers at each step, the content in mathematical and physical knots for each of these subspaces is shown in Table 1. In order to evaluate the improvement in efficiency when GMK is deployed in a Grid environment (namely the EGEE infrastructure), each experiment was split in processes (jobs). Therefore, an experiment is defined by two parameters; the number $k$ of conformations in the subspace, and the number $n$ of processes it is split
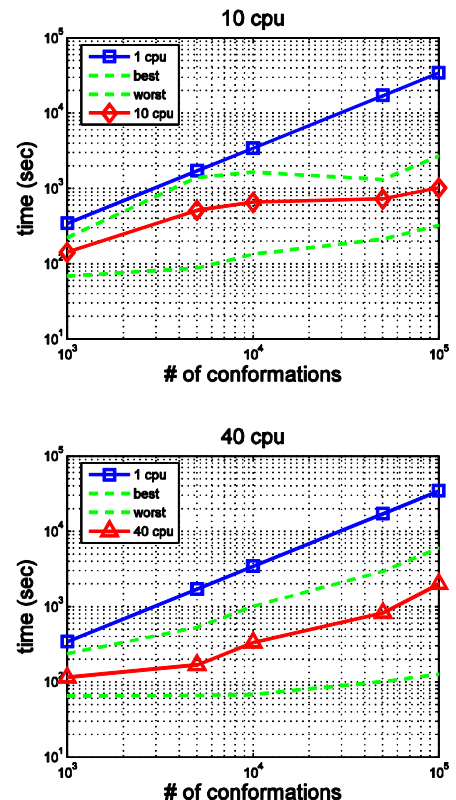

Deleted: doted

into. This procedure determines that each process in an experiment evaluates $k / n$ conformations. Finally, in order to obtain a good representation of the actual time consumption for the procedure, each experiment is repeated 3 times. Early results of this analysis were presented in [37]. The processing time in each case versus the sequential approach is presented in Figure 5.

As demonstrated in the log-log plot diagrams of

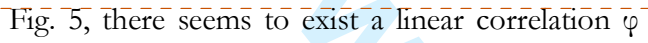
between the execution time $t$ and the number of conformations N. Based on this assumption, it is possible to write:

$$
\ln (\mathrm{t})=\varphi \times \ln (\mathrm{N})=\ln (\mathrm{N} \varphi) \Rightarrow \mathrm{t}=\mathrm{N} \varphi
$$

\section{Formatted: Font: Italic}

Deleted: $\mathrm{h}$
Deleted: 28
Deleted: against

Deleted: several subspaces, containing from 1,000 to 100,000 conformations.

Deleted: Early results of this analysis where presented in [28].

Formatted: Centered

Deleted: 1 (sequential approach), 10, 20 and 40

Formatted: Font: Italic

Formatted: Font: Italic

Formatted: Font: Italic 
Experimentally, in the sequential approach constant $\varphi$ varies from 1.55 to 2.15 . In the gMolKnot approach $\varphi$ takes values from $(1.3-1.5)$ in the worst case and from $(0.35-0.5)$ in the best | case. This translates into a significant

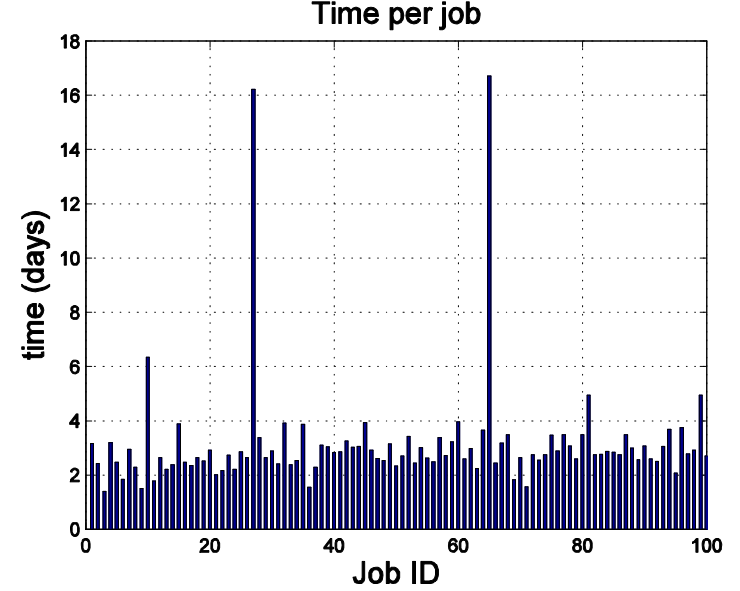

Regarding hardware requirements, worth noting is the following issue: the program, and more specifically the Knot algorithm, was initially designed to run on 32-bit architectures. However, a non-transparent update on the Grid

Fig. 6. Time (in days) that was required for each one of the 100 Jobs (i.e. splits of the conformational space). It is interesting to note that although the average processing time was 3.2 days, the distribution of the execution times is far from uniform. Specifically, the jobs with IDs 26 and 64 took a lot of time, which is explained by the greater yield of knotted conformers in these search areas (as shown in Figure 7).

improvement in the order of the time complexity. There is also a significant gap between best- and worst-case speedups. Moreover, a trend can be observed, where the speedup tends to increase with the number of jobs. Analysis of these initial experiments showed that the cause of the differences in speedup is the heterogeneity of the resources in a Grid environment. Based on this conclusion, the same experiment was performed on a subset of the Grid resources available, namely homogenous resources with CPU Clock over $3,00 \mathrm{GHz}$ and $4 \mathrm{~Gb}$ of RAM. Although there was a substantial improvement in the range of processing times (best / worst / average), especially when 20 or 40 jobs where utilized, the variations in the $\varphi$ value for different number of conformations $\mathrm{N}$ remain. However, this can be attributed to the fact that there is a different content of knotted conformations in each subspace, therefore significantly affecting the processing time (results available on request). infrastructure hardware changed several nodes to 64-bit, thus leading to erroneous results. For this reason, the program has been modified to run without problems on both architectures.

\subsection{Large scale computational experiment}

\subsubsection{Time Efficiency}

The final experiment was split in 100 different processes _(jobs), e each _ exploring_ a _total of 5,538,440 conformations. Figures 6 and 7 show the overall statistics of the $\mathrm{C}_{36} \mathrm{H}_{74}$ run. Specifically, Figure 6 shows the processing time for each of the 100 jobs. Using the simplistic assumption that the sequential processing time would be the sum of the concurrent runs, the speedup measured was approximately 18 (in order to give the magnitude of the calculation, the sequential processing time would be 10.43 months, against the 16.72 days of the worst run). The calculated speedup is much greater if measured against the projected sequential time; based on the experimental results
Deleted: Thus, the effect of the heterogeneity on the efficiency of the procedure is expected to be considerably reduced. 9
Deleted: the large scale experiment was performed on a subset 
on smaller sequential runs of the same length (i.e. 36 carbon atoms in the molecular chain), the average processing time for each conformer is 0.258 seconds. For the total conformational space, this time would lead to a total processing time of 4.6 years and the corresponding speedup would be $\approx 100$.

At this point, it must be noted that the processing time for each conformer through the Mathematics filter is $\approx 0.03$ seconds and $\approx 10.3$ seconds for the Molecular Mechanics filter. Since every conformation must be examined by the Math filter, this step constitutes the actual bottleneck in the algorithm's performance.

Finally, the heterogeneity in the time response, as shown in Figure 6, can be explained by the nature of the actual problem, i.e. the systematic generation of conformers produces dense and sparse areas. These dense areas are responsible for the computational peaks shown in the figure.

\subsubsection{Knotted conformers}

Based on the total output of the g-MolKnot application, the main focus shifted toward the analysis of the 4-crossing knots. Regarding the 3knots, there exist both loose and tight conformations. The difference between "loose" and "tight" lies in the deformation of the molecular chain, and therefore in the energy content of the knotted molecular conformation. The same principle applies in the knots with 4 crossings. However, loose 4-knots require larger segment lengths, and thus a longer molecular chain. For this reason, although the main production of the algorithm has been 3-knots, the
36 carbon atoms in the chain are not enough to create loose 4-knots and the production has been naturally restricted to tight 4-knots (as detected by the molecular mechanics filter).

The main production of tight 4-knots $(\approx 30 \%)$ was delivered by the search areas of job IDs 64 and 26 (as presented in Figure 7) with 129 and 63 4-knots correspondingly. The segment lengths in these areas are dominated by the combinations of 2-6-1-0 and 3-4-2-0 (overall coverage $\approx 72 \%$, where the numbers in each case correspond to lengths of 1, 2, 3 and 4 (i.e. $2-6-$ 1 - 0 stands for " 2 segments with length 1, 6 segments with length 2, 1 segment with length 3 and none with length 4"). It is obvious that the aforementioned combinations represent a much smaller percentage of SubKCS (Eq. 7). This information can be utilized for the redesign of the algorithm in order to make it more efficient for longer molecular chains and, therefore, higher complexity knotted conformations.

At this point, there is the opportunity to discuss the problem of the production of 3- and 4- knots by the Math filter, and the corresponding results of the Molecular Mechanics filter application. Regarding the Math filter, subspace areas (Jobs) with IDs 26 and 64 are rich in knot production (in order of $\approx 10^{5}$, whereas the relatively poor subspace is ID 23 (with a yield $\approx 2 \times 10^{3}$ ). Accordingly, Figure 7 shows that 4-knot production is at a maximum $\left(\approx 2 \times 10^{3}\right)$ in areas 26 and 64 (as discussed previously), and at a minimum in area 23. Finally, the Molecular Mechanics filter displays maximum production of 3 - and 4-knots in the same areas as the Math filter (order of $\approx 2.5 \times 10^{3}$ and 150 respectively). 

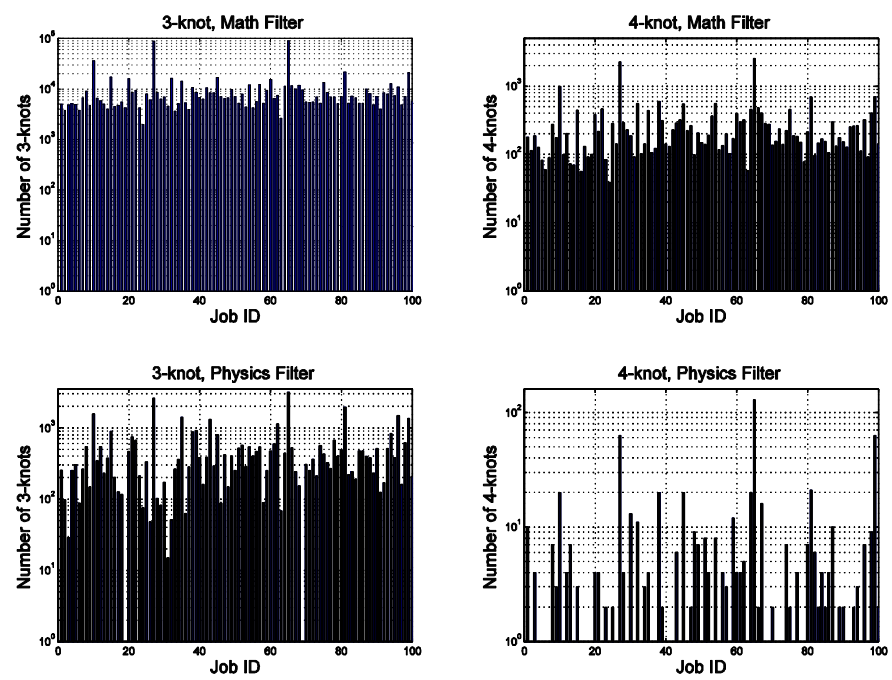

Fig. 7. The top figures present (in logarithmic scale) the production of 3- and 4-crossing knots after the Mathematics filter. The bottom figures present the production of 3- and 4-knots after the molecular mechanics filter.
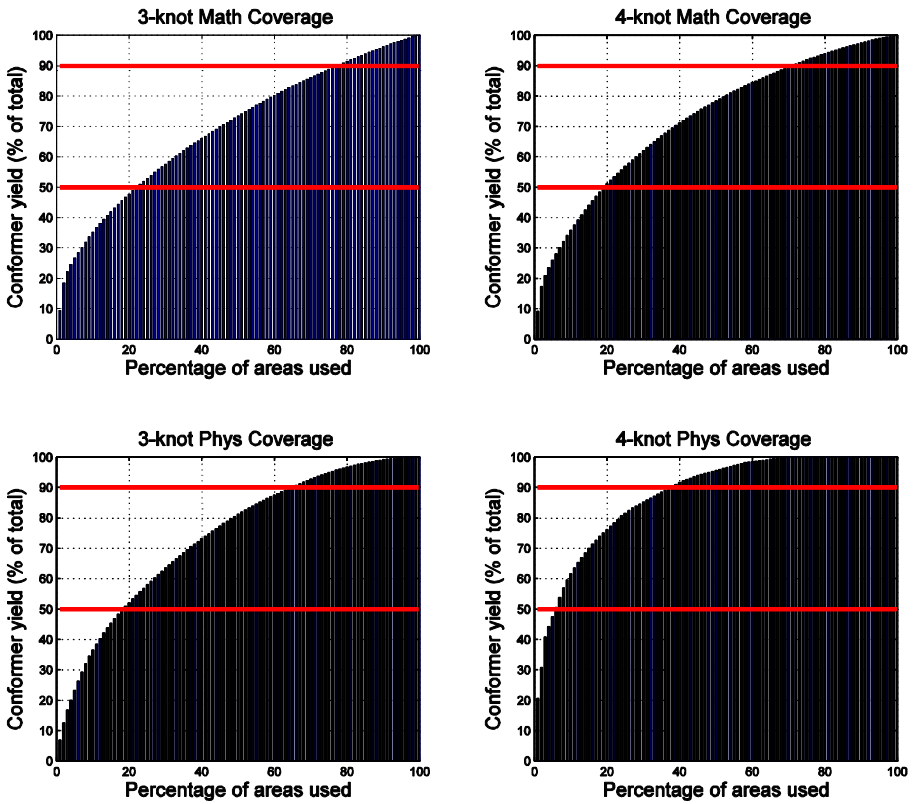

Fig. 8. The diagrams present the yield in 3-and 4-knots for each of the filters. This is achieved by re-ordering the jobs by their yield in conformers in descending order. Thus, for example, $20 \%$ of the total jobs (computational time) produces approximately $75 \%$ of the 4 -knots in the molecular mechanics filter.

Ordering the subspaces by production of 3- and 
4-knots, as shown in Figure 8, it is obvious that approximately $20 \%$ of the jobs are enough to produce almost $50 \%$ of the knots, regarding the mathematics filter. For the molecular mechanics filter, and especially for 4 -knots, less than $10 \%$ of the jobs can produce_more than 50\%_of the final knots. This analysis shows that there exist combinations of jine segments lengths with greater propensity to creating knots, Indeed, the approach is also verified by the mathematical theorems proposed by Cantarella_et al [19] and Clark et al [20], who studied the potential of polygonal lines consisting of 5 and 6 segments to create knots. For instance Clark et al [20] proved that Figure-Eight knot should satisfy the following inequalities (see Fig. 3):

$$
\begin{aligned}
& \mathrm{L}_{1} \geq \mathrm{L}_{2}+\mathrm{L}_{3}+\mathrm{L}_{4}+\mathrm{L}_{5} \\
& \mathrm{~L}_{6} \geq \mathrm{L}_{2}+\mathrm{L}_{3}+\mathrm{L}_{4}+\mathrm{L}_{5}
\end{aligned}
$$

$\underline{\text { So instead of building all combinations, in order }}$ to reduce heterogeneity, we could enter conformers that comply with the 3.6 Theorem [20] for the case of 6 straight segments. Future mathematical analysis would provide us with insights for knotted conformers of more than 6 line segments.

This test shows that with the appropriate posteriori analysis of the possibilities to represent the polygonal line while keeping constant the total length and varying the segments and their relative positions, it is possible to detect the combinations of the segments in order to produce $50 \%$ of the total results with only $20 \%$ of the initial | computational cost. This analysis would aim to

Table 2

Total yield of conformers after each filter, according to knot complexity (3- and 4- crossings).

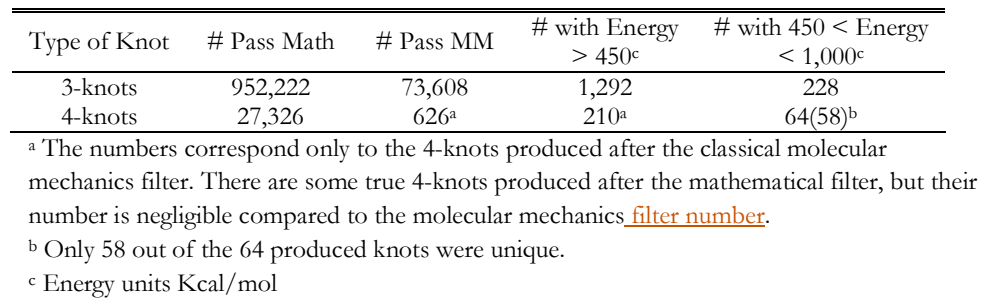

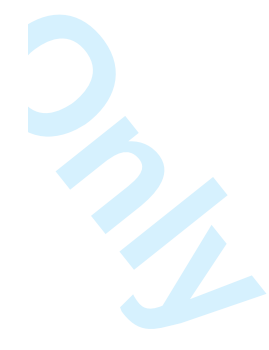

Figure $7^{-}$shows the number of 3 -knots ${ }^{-}$and $^{-} 4-$ knōts ${ }^{-}$that ${ }^{-} \overline{p a s s e d}^{-}$the $^{-}$Mathematics ${ }^{-}$and ${ }^{-}$the $^{-}$ Molecular Mechanics filter. Looking at the actual

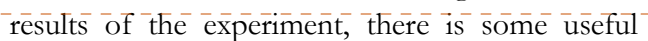
information regarding the yield of 3-knots and 4knots. This information is summarized in Table 2. $\underline{\text { Krinas et al [27] showed that the knotted energy is }}$ spreading all over the knotted region of the molecule and induces distortion in bond lengths, bond angles and torsion angles; furthermore a mathematical expression of the energy partition is -presented. - There is-no relationship between thenumber of crossings and the knotted energy. However, it would be possible to derive one once 5-knots and 6-knots are produced. Moreover, a quantitative structure-activity relationship could emerge by measuring the ability of tight knots to act as mediators in reactions (catalysts).

From Table 2, and given that the size of SubKCS is $553,844,736$, approximately $0.2 \%$ of the conformers are 3- and 4-knots detected by the Math filter. Moreover, the analysis of the results showed that there exists a possibility for further improvement, if the subspaces that are much more efficient in knot production than others can be identified. Therefore, the analysis of all subspaces, similar to the analysis of the 4-knots

\begin{tabular}{l} 
Deleted: the approach that \\
Deleted: lengths \\
Deleted: , is correct \\
Deleted: \\
Deleted: 4 \\
\hline
\end{tabular}

\begin{tabular}{|l|}
\hline Formatted: Subscript \\
\hline Formatted: Subscript \\
\hline Formatted: Subscript \\
\hline Formatted: Subscript \\
\hline Formatted: Subscript \\
\hline Formatted: Centered \\
\hline Formatted: Subscript \\
\hline Formatted: Subscript \\
\hline Formatted: Subscript \\
\hline Formatted: Subscript \\
\hline Formatted: Subscript \\
\hline
\end{tabular}

Deleted: produced 
discussed previously, is expected to allow for segment length combinations which will improve the overall efficiency of the MolKnot algorithm, possibly by one or even two orders of magnitude.

Finally, the sizable difference between the processing times of the Math and the Molecular Mechanics filters (approximately 0.03 seconds vs 10.3 seconds) suggests that improvements can be made regarding the load-balancing of the procedure. The GMK is essentially a modular algorithm as far as the two filters are concerned, hence functional decomposition can be achieved. A minor restructuring of the algorithm may allow for the step-by-step execution of the program, where the output of each step is uniformly distributed in the concurrent processes of the next one.

\section{Conclusions}

This paper has described the development and analysis of the grid version of the MolKnot program and has shown how it has used to obtain for the first time complex structures as open molecular 41 knotted $\mathrm{C}_{36} \mathrm{H}_{74}$ conformers. During this process it has been shown that:

| the algorithm can be transferred to the grid, due to its embarrassingly parallel nature

- the complexity analysis of both GMK and gMolKnot algorithms has determined that the Math filter is the most CPU consuming process, and the final complexity is $\mathrm{O}(n c, N, p)=\frac{\ln ^{\frac{2}{3}}(N) \times O\left(n c^{3}\right)}{p}$

- the $\mathrm{C}_{36} \mathrm{H}_{74}$ test case shows that the yield of the actual knot conformers is definitely not uniform across the different_jobs

There is no doubt that there exists an increasing interest for complex (with 5 or more crossings) molecular structures, which exhibit several interesting properties. However, the computational cost, as the actual molecular chain lengths get longer, becomes prohibitively large. An estimate of the minimum number of carbons involved in a knot formation is 30 for a 4 -crossing knot, 38 for 5 -crossing knots, and 44 for the 6 crossing knots. Although this hypothesis needs further testing, these estimates lead to enormous conformational spaces, over a trillion in number. Future work should target the optimization of the g-MolKnot code in order to make it more efficient and cost-effective for longer molecular chains. The next milestone should be the detection of 5crossing knots.

An additional issue that requires further study is the heterogeneity in time efficiency, due to the nature of the problem as discussed in Section 3.3.1. Adaptive loading techniques may provide satisfactory solutions to this problem.

In the end, the adaptation of the MolKnot code to a Grid environment has enabled the detection of interesting structures with 4-crossings and has provided an analysis framework for more efficient searching of knots in longer molecular chains.

\section{Acknowledgements}

The research Project is co-funded by the European Union - European Social Fund (ESF) \& National Sources, in the framework of the program "HRAKLEITOS" of the "Operational Program for Education and Initial Vocational Training" of the 3rd Community Support Framework of the Hellenic Ministry of Education. The authors gratefully acknowledge the computer time provided by the Hellas Grid infrastructure and the computer time provided by the Research Center for Scientific Simulations (RCSS) of Ioannina University, Greece. The following software packages have been used: Knot [38], Tinker [39], Gamess-US [40]

\section{Appendix A}

Figure 3(b) Molecular segments used for the assembly of the initial molecular geometry $\left(\mathrm{L}_{i}\right.$,
Deleted: transfered

Field Code Changed 
black color), ( $\mathrm{B}_{\mathrm{j}}$, red color) with: $\mathrm{L}_{1}=\Phi_{1}, \mathrm{~B}_{1}=$ $\Phi_{2} \Phi_{3}, \mathrm{~L}_{2}=\Phi_{4}, \mathrm{~B}_{2}=\Phi_{5} \Phi_{6}, \mathrm{~L}_{3}=\Phi_{7} \Phi_{8}, \mathrm{~B}_{3}=$ $\Phi_{9} \Phi_{10}, \mathrm{~L}_{4}=\Phi_{11} \Phi_{12}, \mathrm{~B}_{4}=\Phi_{13} \Phi_{14}, \mathrm{~L}_{5}=\Phi_{15} \Phi_{16}$, $\mathrm{B}_{5}=\Phi_{17} \Phi_{18}, \mathrm{~L}_{6}=\Phi_{19} \Phi_{20} \Phi_{21}, \mathrm{~B}_{6}=\Phi_{22} \Phi_{23}, \mathrm{~L}_{7}=$ $\Phi_{24}, \mathrm{~B}_{7}=\Phi_{25} \Phi_{26}, \mathrm{~L}_{8}=\Phi_{27} \Phi_{28}, \mathrm{~B}_{8}=\Phi_{29} \Phi_{30}$ and $\mathrm{L}_{9}=\Phi_{31} \Phi_{32} \Phi_{33}$ where $\Phi_{1}, \Phi_{2}, . ., \Phi_{33}$ are CCCC torsion angles in the carbon backbone of the $\mathrm{C}_{36} \mathrm{H}_{74}$ molecule.

Figure 3(c) The initial building sequence $\Phi_{1}, \Phi_{2}, .$. , $\Phi_{33}$ is: $180^{\circ},+95^{\circ},-60^{\circ}, 180^{\circ},-95^{\circ},+60^{\circ}, 180^{\circ}$, $180^{\circ},+95^{\circ},-60^{\circ}, 180^{\circ}, 180^{\circ},+95^{\circ},-60^{\circ}, 180^{\circ}$, $180^{\circ},-60^{\circ},+95^{\circ}, 180^{\circ}, 180^{\circ}, 180^{\circ},+60^{\circ},-95^{\circ}$, $180^{\circ},-95^{\circ},+60^{\circ}, 180^{\circ}, 180^{\circ},-95^{\circ},+60^{\circ}, 180^{\circ}$, $180^{\circ}, 180^{\circ}$.

Figure 3(d) Tube representation of the outcome of the geometry optimization procedure of the $\mathrm{C}_{36} \mathrm{H}_{74}$ hydrocarbon; the sequence of CCCC backbone torsion angles (at B3LYP level): $-174.6^{\circ}, 133.1^{\circ}, 38.1^{\circ},-5.9^{\circ}, 58.7^{\circ},-99.0^{\circ}, 132.5^{\circ}$, $-133.9^{\circ}, 176.2^{\circ},-130.5^{\circ}, 142.6^{\circ},-137.1^{\circ}, 127.7^{\circ}$, $-53.1^{\circ},-81.1^{\circ}, 91.9^{\circ},-65.6^{\circ}, 84.8^{\circ},-122.4 .0^{\circ}$, $-103.6^{\circ}, 50.2^{\circ}, \quad 58.7^{\circ},-92.9^{\circ}, 111.7^{\circ},-95.1^{\circ}$, $115.8^{\circ},-127.8^{\circ}, 129.0^{\circ},-163.9^{\circ}, 109.6^{\circ},-172.9^{\circ}$, $56.4^{\circ},-161.6^{\circ}$ (hydrogens are not shown for clarity). CC bonds (BL) are in the range $1.538 \AA \leq$ $\mathrm{BL} \leq 1.685 \AA$ and $\mathrm{CCC}$ bond angles $(\mathrm{BA})$, are between 109.3 $\leq \mathrm{BA} \leq 145.0^{\circ}$ (B3LYP/6-31G** optimized geometry. Frequency calculations confirmed the local minimum at the same level of theory).

\section{References}

[1] O. Lukin and F. Vögtle, Knotting and threading of molecules: chemistry and chirality of molecular knots and their assemblies, Angewandte Chemie International Edition 44 (2005) 14561477

[2] C. Liang and K. Mislow, Knots in proteins, Journal of American Chemical Society 116 (1994) 11189-11190.

[3] W. R. Taylor, A deeply knotted protein structure and how it might bold, Nature 406 (2000) 916-919.
[4] L. F. Liu, R. E. Depew and J. C. Wang, Knotted singlestranded DNA rings: A novel topological isomer of circular singlestranded DNA formed by treatment with Escherichia coli $\omega$ protein, Journal of Molecular Biology 106 (1976), 439.

[5] H. Adams, E. Ashworth, G. A. Breault, J. Guo, C. A. Hunter and P. C. Mayers, Knot tied around an octabedral metal centre, Nature, 411 (2001) 763.

[6] V. Balzani, A. Credi, F. M. Raymo and J. F. Stoddart, Artificial Molecular Machines, Angewandte Chemie International Edition 39 (2000) 3348.

[7] O. Lukin, T. Kubota, Y. Okamoto, F. Schelhase, A. Yoneva, W. M. Müller, U. Müller and F. Vögtle, KnotaxanesRotaxanes with Knots as Stoppers, Angewandte Chemie International Edition 42 (2003) 4542-4545.

[8] F. M. Raymo and J. F. Stoddart, Organic Template-Directed Syntheses of Catenanes, Rotaxanes, and Knots, in: J.-P. Sauvage and C. Dietrich-Buchecker (Eds.), Molecular Catenanes, Rotaxanes and Knots, Wiley-VCH, Weinheim, Germany, 1999, pp. 143-176.

[9] G. Bottari, F. Dehez,D.A. Leigh,P. J.Nash, E. M. P'erez, J. K. Y. Wong and F. Zerbetto, Entropy-Driven Translational Isomerism: A Tristable Molecular Shuttle, Angewandte Chemie International Edition 42 (2003) 5886.

[10] D. J. Craik, M. Čemažar and N.L. Daly, The cyclotides and related macrocyclic peptides as scaffolds in drug design, Current Opinion in Drug Discovery and Development 9 (2006) 251260.

[11] E. K. Wilson, Chemists turned visionaries, Chemical and _Engineering News 78 (2000) 32-45.

[12] A. V. Vologodskiir, A. V. Lukashin, M. D. FrankKamenetskii, and V. V. Anshelevich, The knot problem in statistical mechanics of polymer chains, Pisma $\mathrm{v}$ Zhurnal Eksperimentalnoi i Teoreticheskoi Fiziki 66 (1974), 2153. Soviet Physics Journal of Experimental and Theoretical Physics Letters (JETP Letters), 39 (1974), 1059].

[13] K. Koniaris and M. Muthukumar, Knottedness in ring polymers, Physical Review Letters 66 (1991) 2211.

[14] A. M. Saitta, P. D. Soper, E. Wasserman, M. L. Klein, Influence of a Knot on the Strength of a Polymer Strand, Nature 399 (1999) 46.

[15] P. Virnau, Y. Kantor, M. Kardar, Knots in Globule and Coil Phases of a Model Polyetbylene, Journal of American Chemical Society 127 (2005) 15102.

[16] K. Millet, A. Dobay, A. Stasiak, Linear Random Knots and Their Scaling Behavior, Macromolecules 38 (2005) 601.
Formatted: Superscript

Formatted: Superscript

Formatted: Font: Italic

Formatted: Font: Italic

Formatted: Font: Italic

Formatted: Font: Italic

Formatted: Font: Italic 
[17] C. S.Krinas and I. N. Demetropoulos, A systematic algoritbm capable to yield open molecular knots: Application to alkanes, polyethylene oxides and peptides, Chemical Physics Letters 433 (2007) 422-426.

[18] Generalized Molecular Knotting Algorithm, http://users.uoi.gr/me00599/index.html

[1기 J. Cantarella and H. Johnston, Nontrivial embeddings of polygonal intervals and unknots in 3-space, Journal of Knot Theory and Its Ramifications 7 (1998) 1027-1039.

[20] T. J. Clark and G. A. Venema, Classifying polygonal chains of six segments, Journal of Knot Theory and Its Ramifications 13 (2004) 479-514.

| 21] D. Bonchev and D. H. Rouvray, Chemical Graph Theory: Introduction and Fundamentals, Gordon and Breach Science Publishers, 1990.

[22] Maria Tasioula-Margari and Ioannis N. Demetropoulos, Viscosity-structure relationship of dilute triglycerides' solutions correlation to retention time in reversed-phase liquid cbromatography. Lournal of the American Oil Chemists' Society 69 (1992) $\underline{1112 .}$

[23] A.C. Wallace, R.A Laskowski, J.M. Thornton, Derivation of $3 D$ coordinate templates for searching structural databases: application to the SerZHisZAsp catalytic triads of the serine proteinases and lipases, Protein Science, 5, 1001 (1996).

[24] V. A. Tatsis, A. Stavrakoudis, I. N. Demetropoulos, LysinebasedTrypsin ActSite(LysT AS): A configurational tool of the TINKER software to evaluate Lysine based branched gyclic peptides as potential chymotrypsin-mimetics, Molecular Simulation 32 (2006) $\underline{643 .}$

[25] F. M. Menger. An alternative view of the ensyme catalysis, Pure and Applied Chemistry 77 (2005) 1873.

[2G J.W. Alexander, Topological invariants of knots and links, Transactions of the American Mathematical Society 30 (1928) 275-306.

[27] C. S. Krinas and I. N. Demetropoulos, $\mathrm{C}_{22} \mathrm{H}_{46}$ : The Smallest Open 31 Knotted Alkane by Computer-Aided Design, Journal of Molecular Graphics and Modelling 26(7) (2008), 1153-1159.

| [28] L. Philpott, Pocket guide to Knots, New Holland Publishers, London UK, 2006.

| [29] M. Delbrück, Knotting problems in biology, in Proceedings of the Symposium of Applied Mathematics, 14 (1962), 55-63.

[30] I. Foster and C. Kesselman, The Grid: Blueprint for a New Computing Infrastructure, Morgan Kaufmann Publishers, 1998.
[31] Enabling Grids for E-sciencE, http://www.eu-egee.org

[32] Uniform Interface to Computing Resources, http://www.unicore.eu/

[33] The Globus Alliance, http://www.globus.org/

[34] Research Center For Grid and Service Computing, http://vega.ict.ac.cn/

[35] H. E. Polychroniadou, F. E. Psomopoulos and P. A. Mitkas, G-Class: A Divide and Conquer Application for Grid Protein Classification, in Proceedings of the 2nd ADMKD 2006: Workshop on Data Mining and Knowledge Discovery (in conjunction with ADBIS 2006: The 10th East-European Conference on Advances in Databases and Information Systems, 2006, pp. 121-132.

[3] D. Coppersmith and S. Winograd, Matrix multiplication via arithmetic progressions, Journal of Symbolic Computation 9 (1990) 251-280.

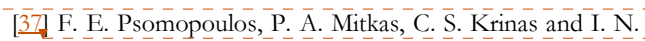
Demetropoulos, G-MolKnot: A grid enabled systematic algorithm to produce open molecular knots, presented at the 1st HellasGrid User Forum, 2008.

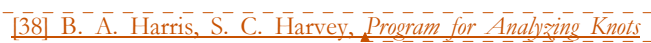
Represented by Polygonal Paths, Journal of Computational Chemistry 20 (1999) 813.

[39] I. W. Ponder, F. M. Richards, An efficient Newton-like method for molecular mechanics energy minimization of large molecules, Lournal of Computational Čhemistry 8 (1987) $10 \overline{1} 6$.

[40] M. W. Schmidt, K. K. Baldridge, J. A. Boatz, S. T. Elber, M. S. Gordon, J. H. Jensen, S. Koseki, N. Matsunaga, K. A. Nguygen, S. Su, T. L. Windus, M. Dupuis, J. A. Montgomery, Generic atomic and molecular electronic structure system, Journal of Computational Chemistry 14 (1993) 1347.

\begin{tabular}{|l|}
\hline Deleted: 22 \\
Deleted: 2 \\
\hline Deleted: 23 \\
\hline Deleted: 24 \\
Deleted: 3 \\
\hline Deleted: 25 \\
\hline Deleted: 4 \\
\hline
\end{tabular}

Deleted: 15

Deleted: 16

Deleted: 27

Formatted: Font: Italic

Deleted: 28

Formatted: Font: Italic Formatted: Font: Italic

Formatted: Font: Italic

Formatted: Font: Italic

Formatted: Font: Italic

Formatted: Font: Italic

Deleted: 17

Deleted: 18

Deleted: 19

Deleted: 0

Deleted: 21 


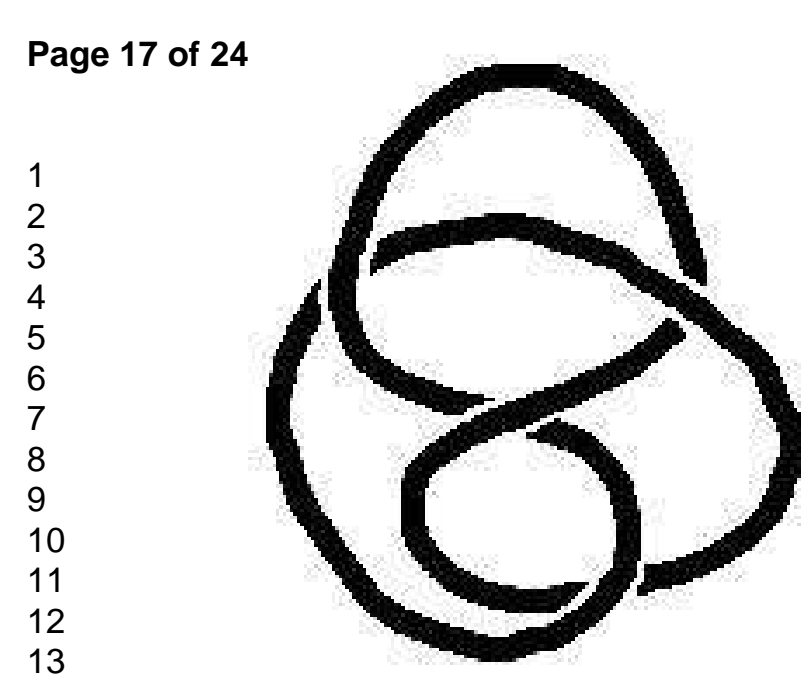

[a]
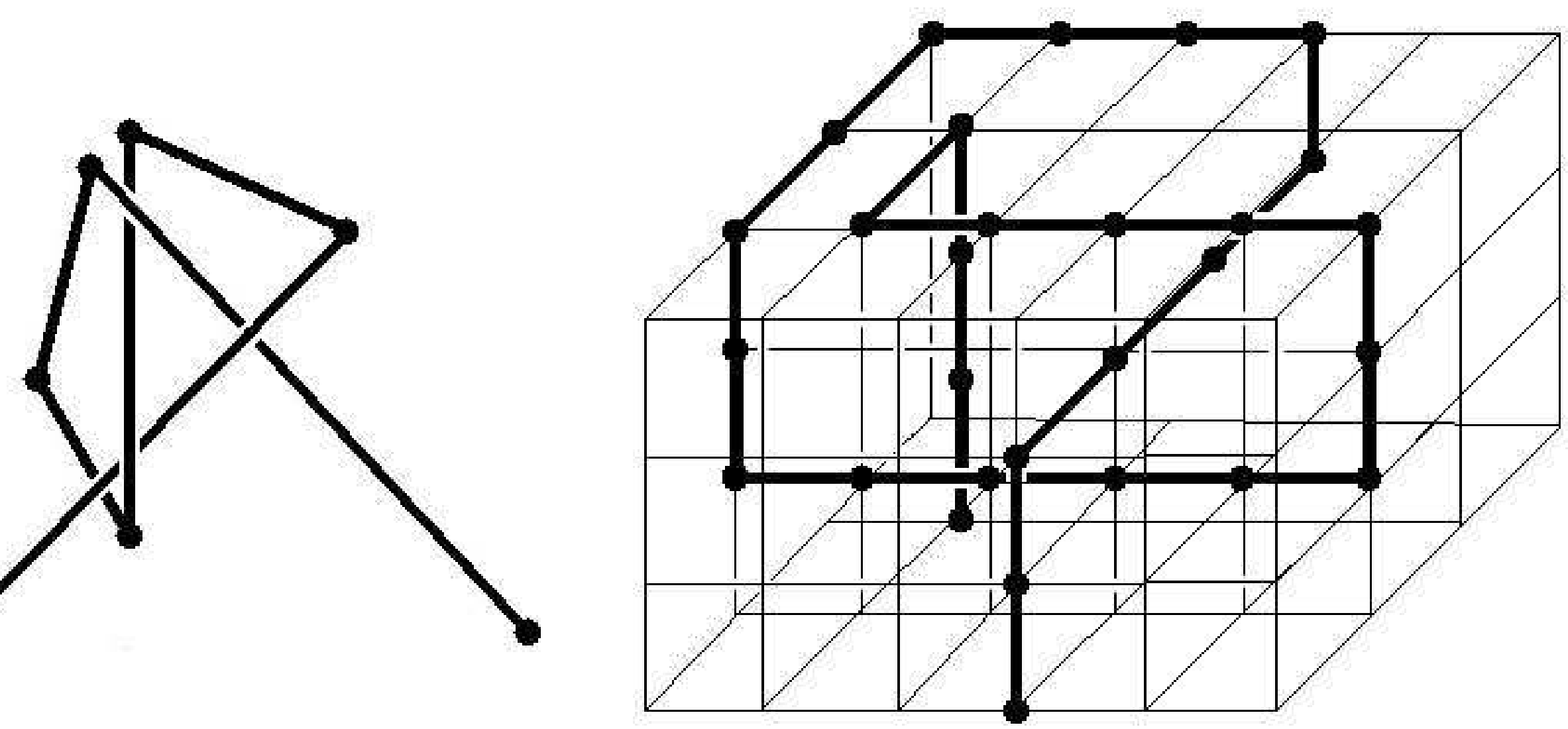

[d]

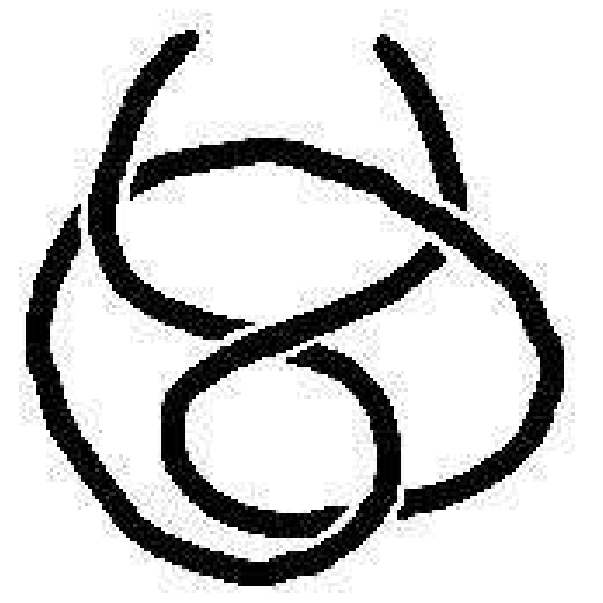

[b]

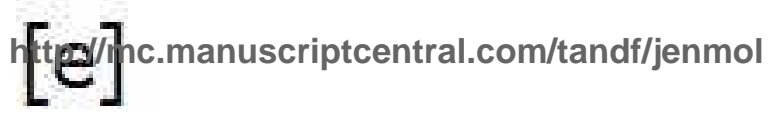

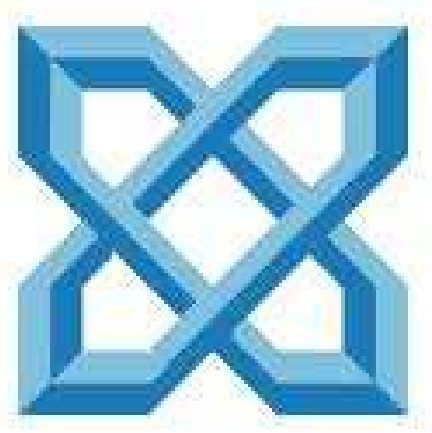

[c]

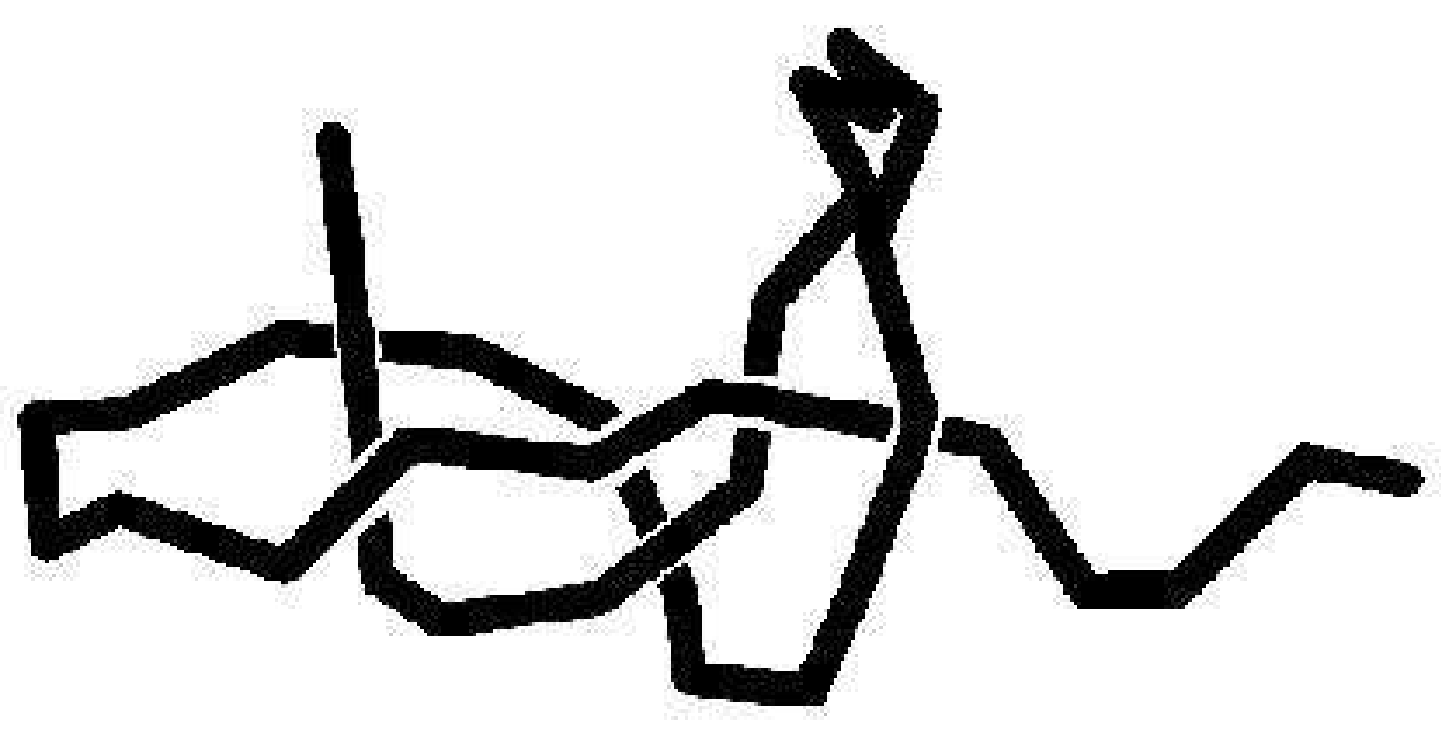

[f] 

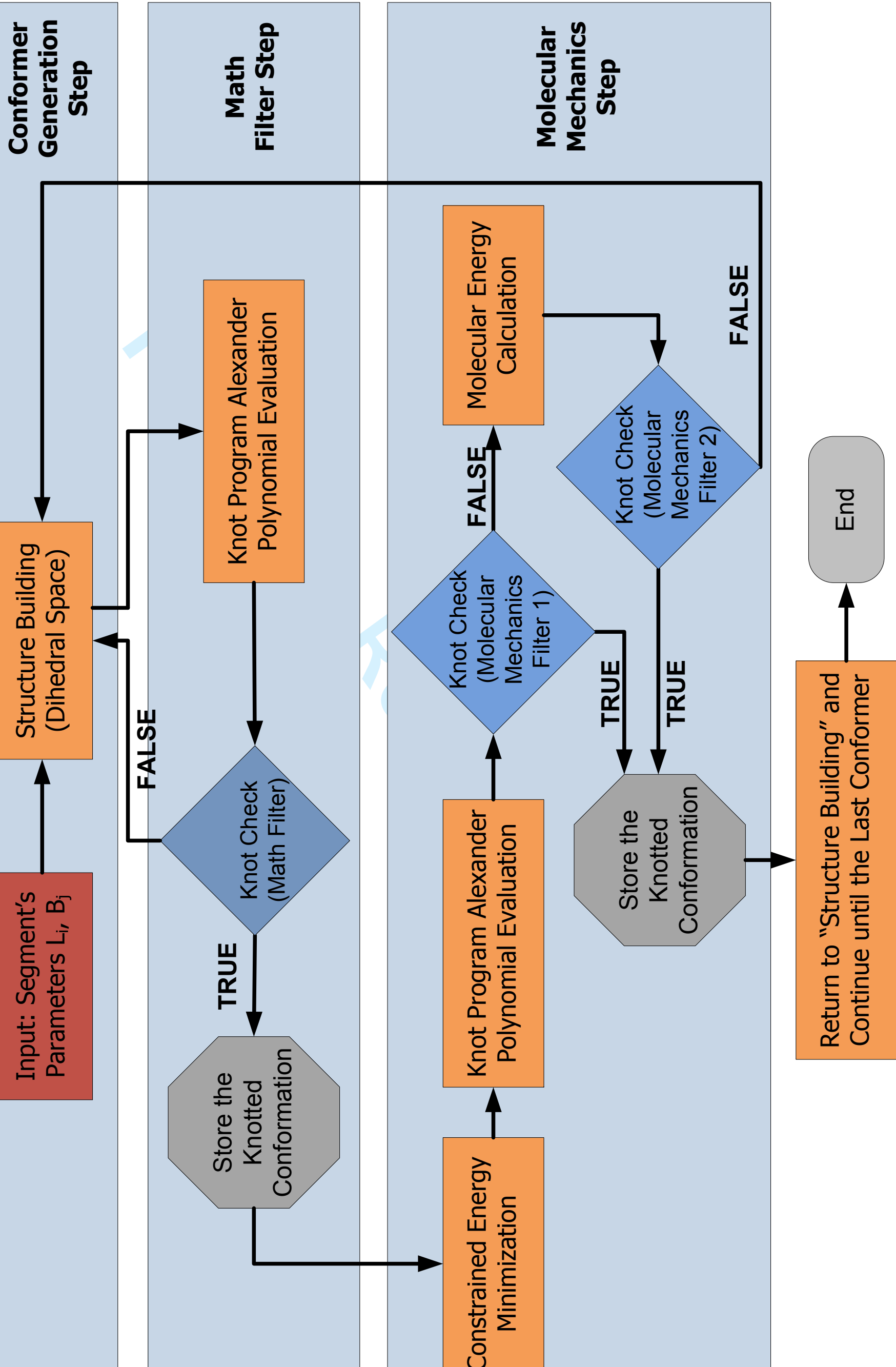
a)

Page 19 of 24

1
2
3

b) 4 $\mathbf{L}_{3}$

15

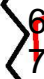

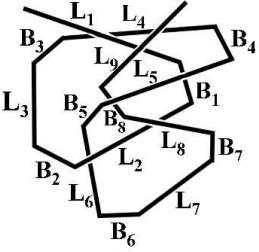

$\mathbf{L}_{\mathrm{d}}^{8}$

$\begin{array}{llllllllllll}\mathbf{L}_{d}^{8} & B_{1} & \mathbf{L}_{2} & \mathbf{B}_{2} & \mathbf{L}_{3} & \mathbf{B}_{3} & \mathbf{L}_{4} & \mathbf{B}_{4} & \mathbf{L}_{5} & \mathbf{B}_{5} & \mathbf{L}_{6} & \mathbf{B}_{6}\end{array}$

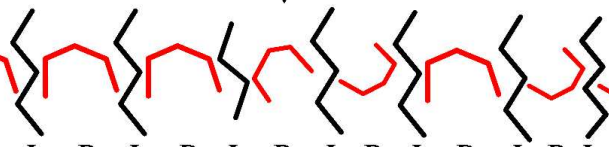

$\checkmark$

10 Nano-Assembly

c) 11

tipo: 1 d)

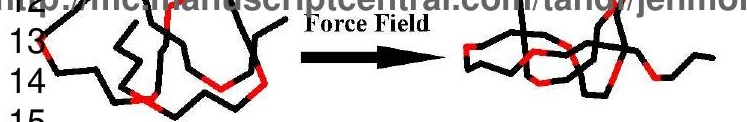




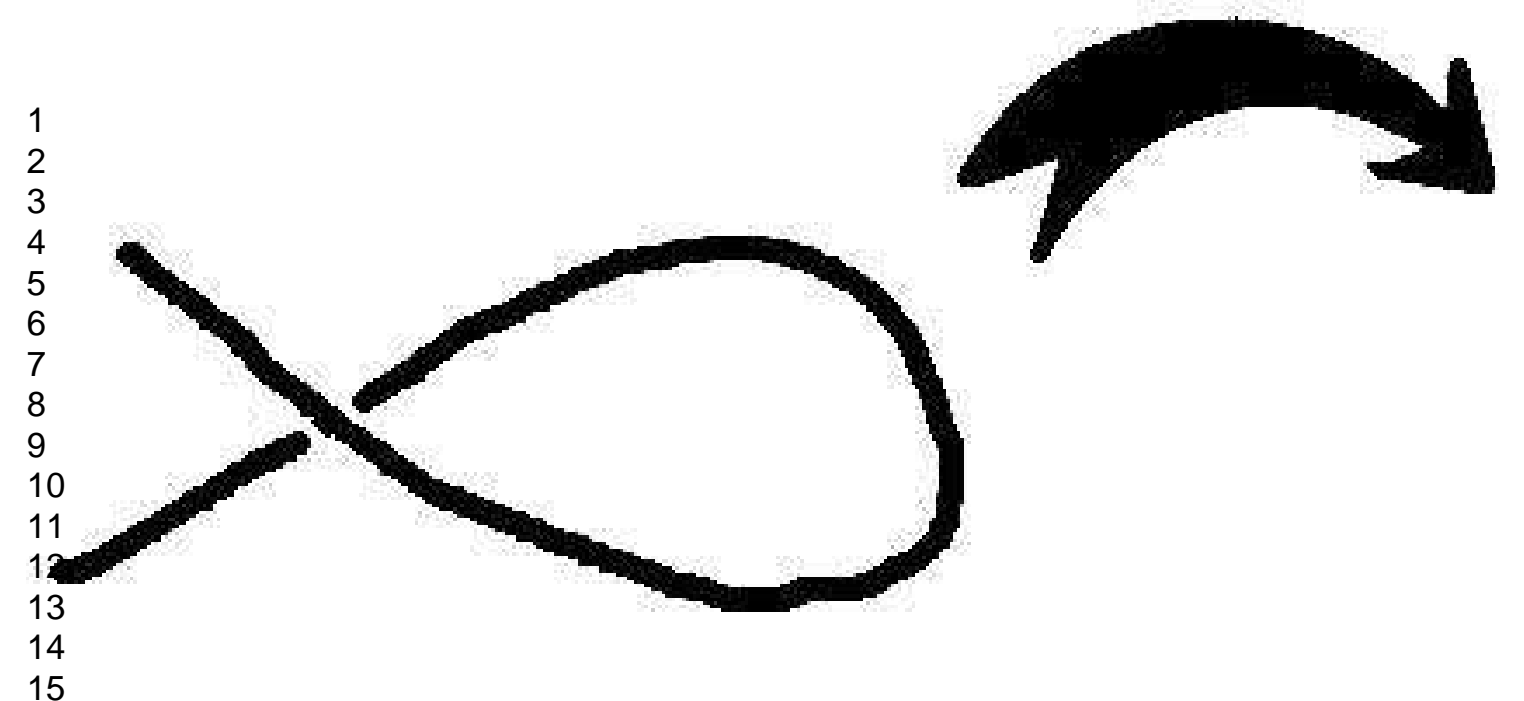

[a]

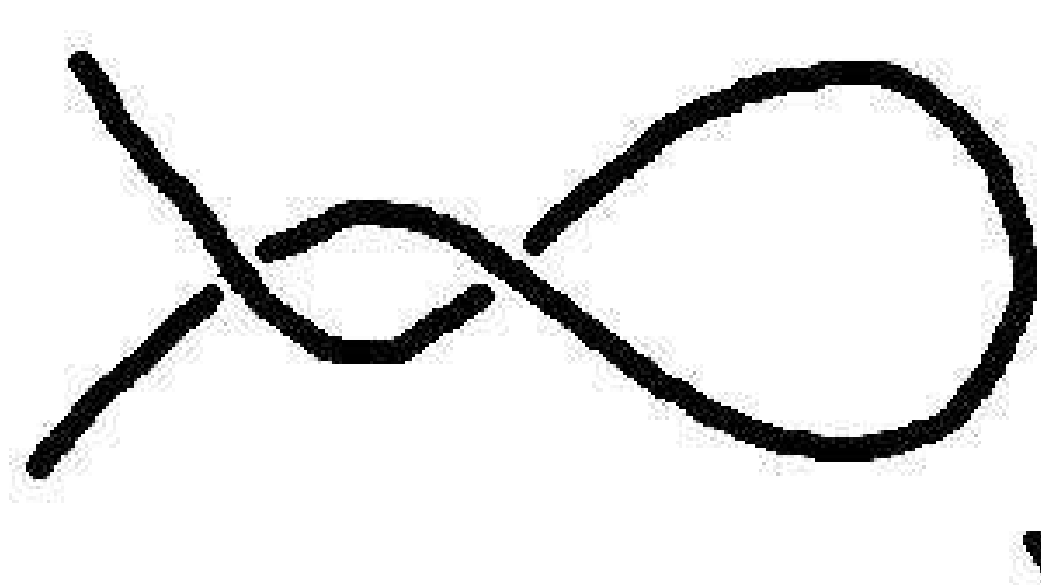

[b]

1

[c]

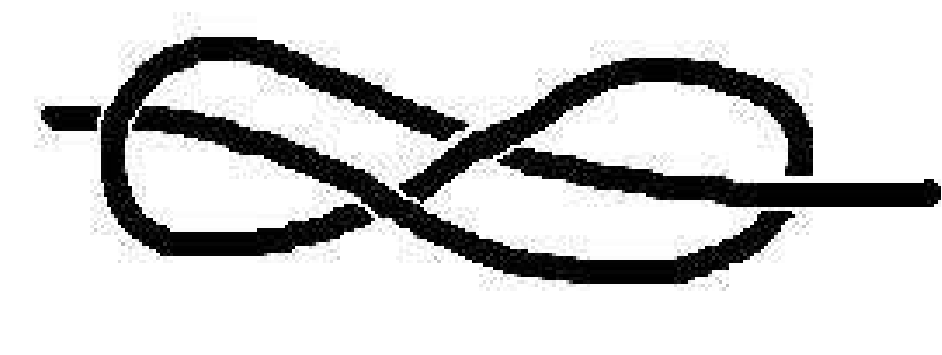

[d] 

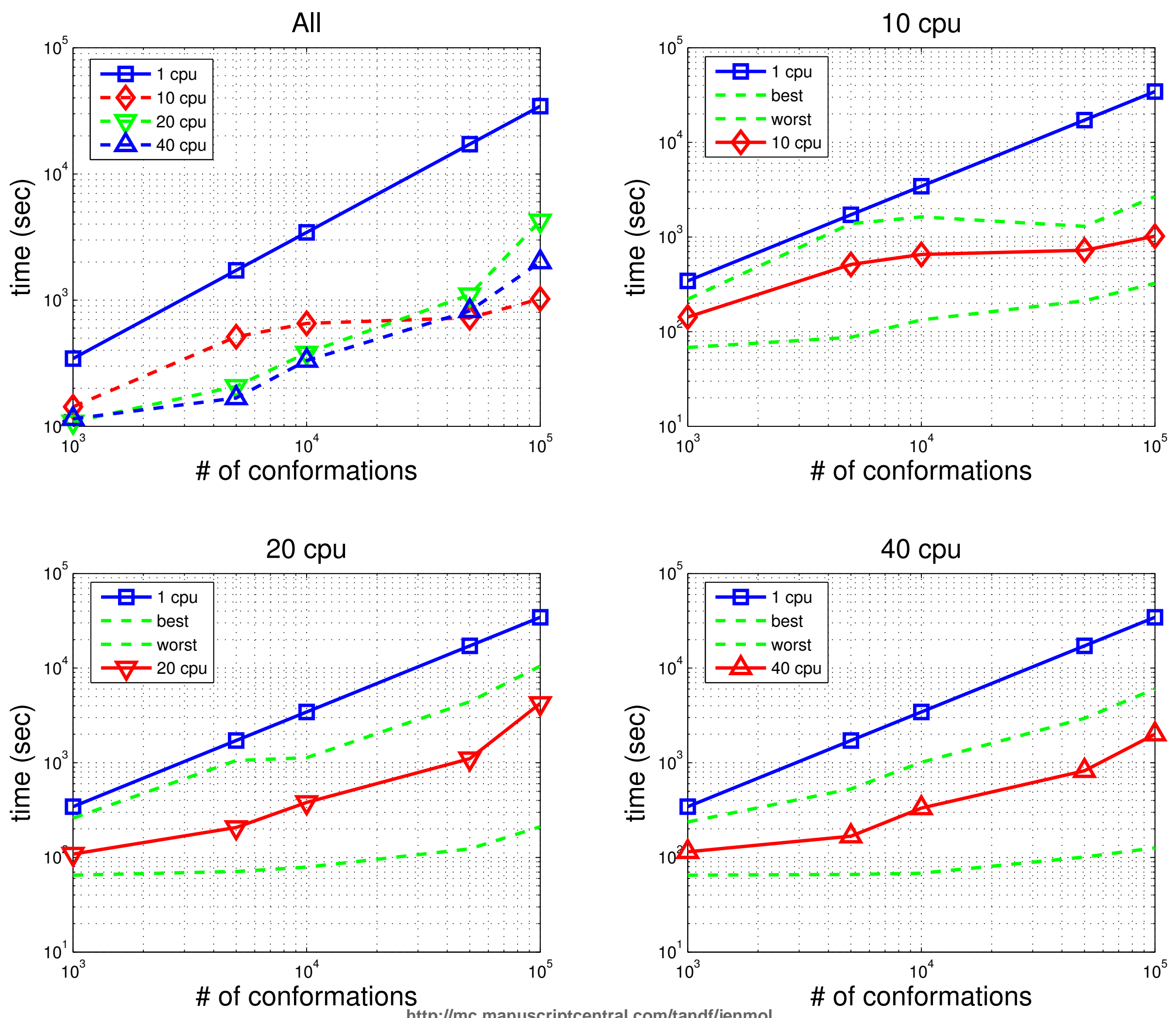

http://mc.manuscriptcentral.com/tandf/jenmol 
Time per job

Page 22 of 24

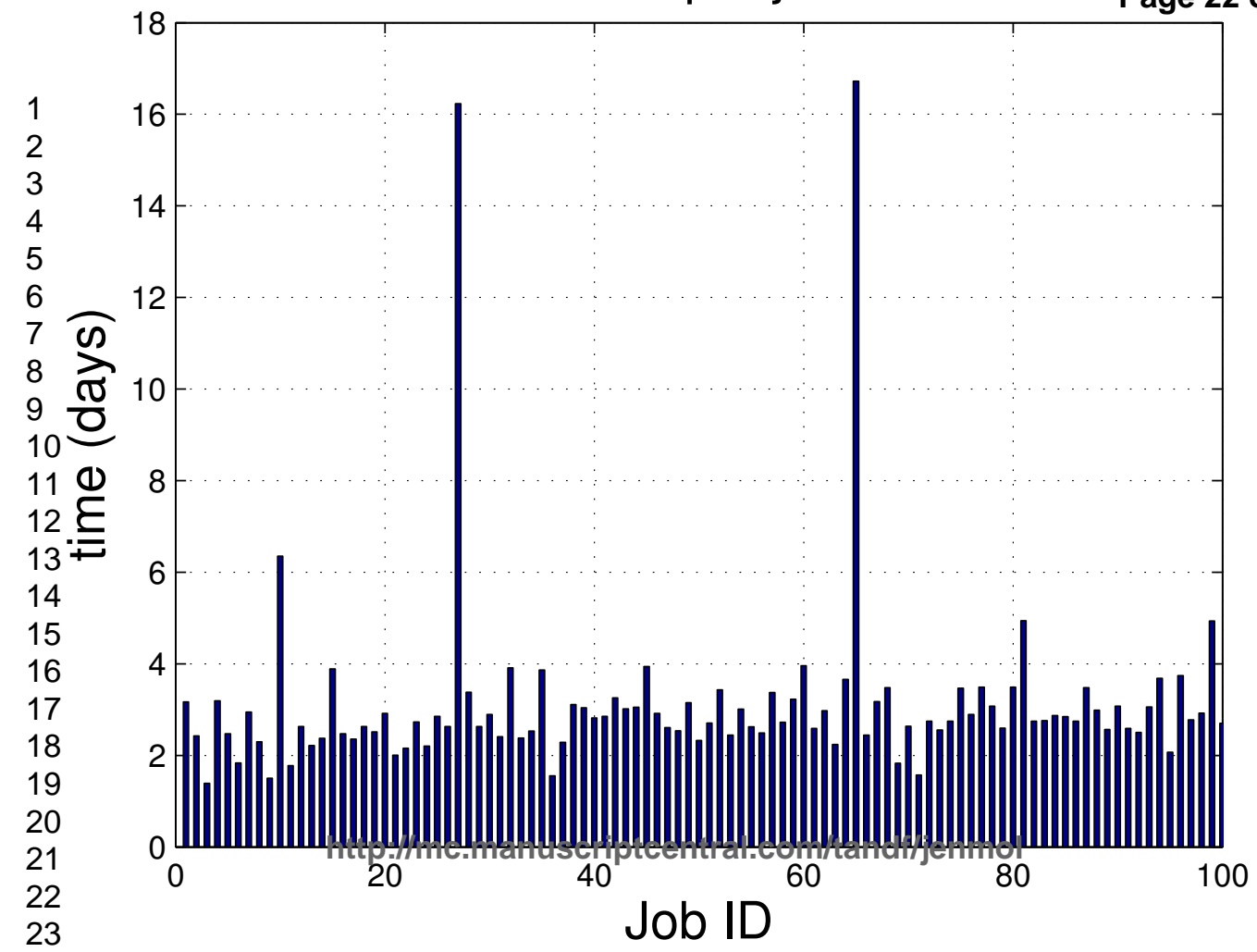



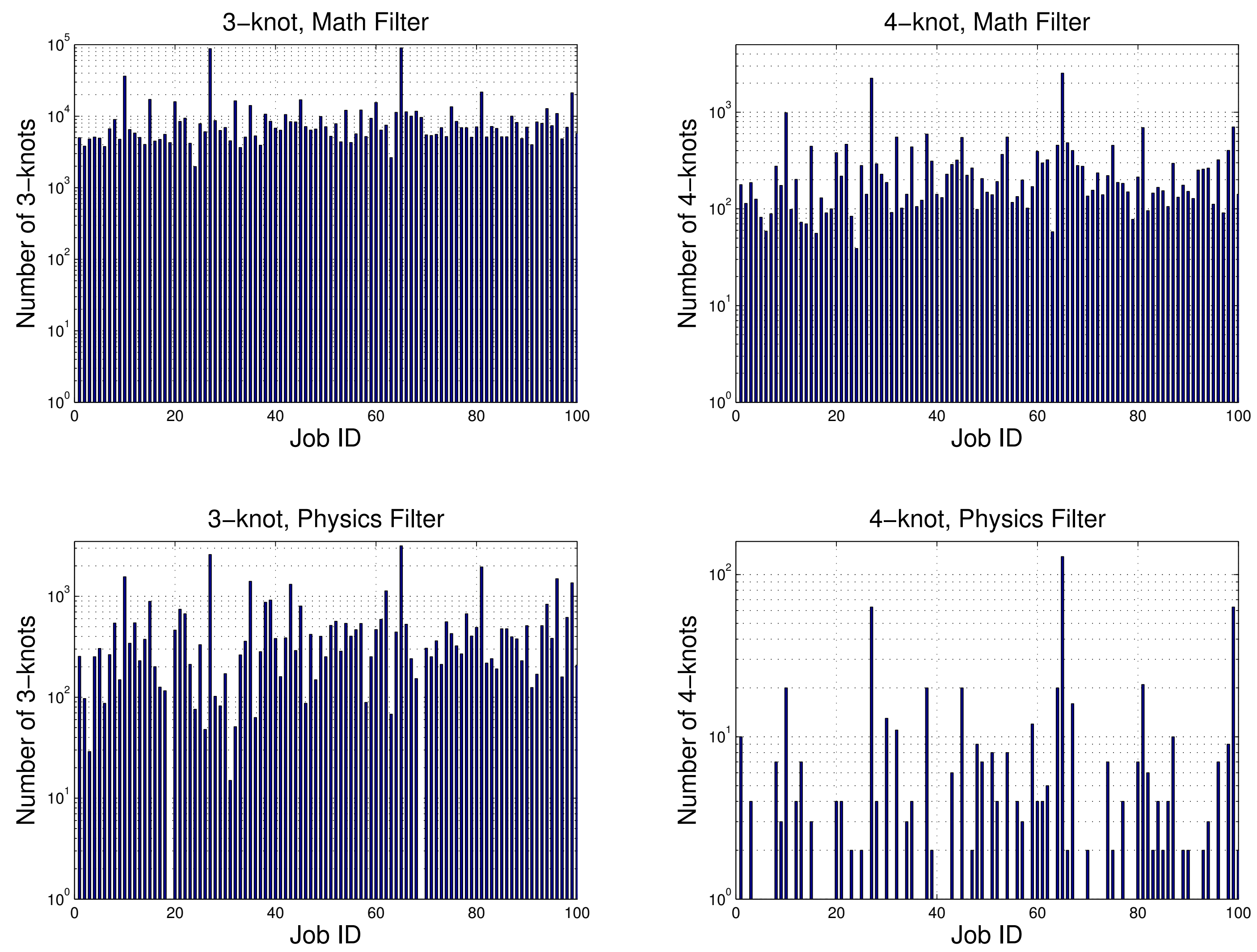

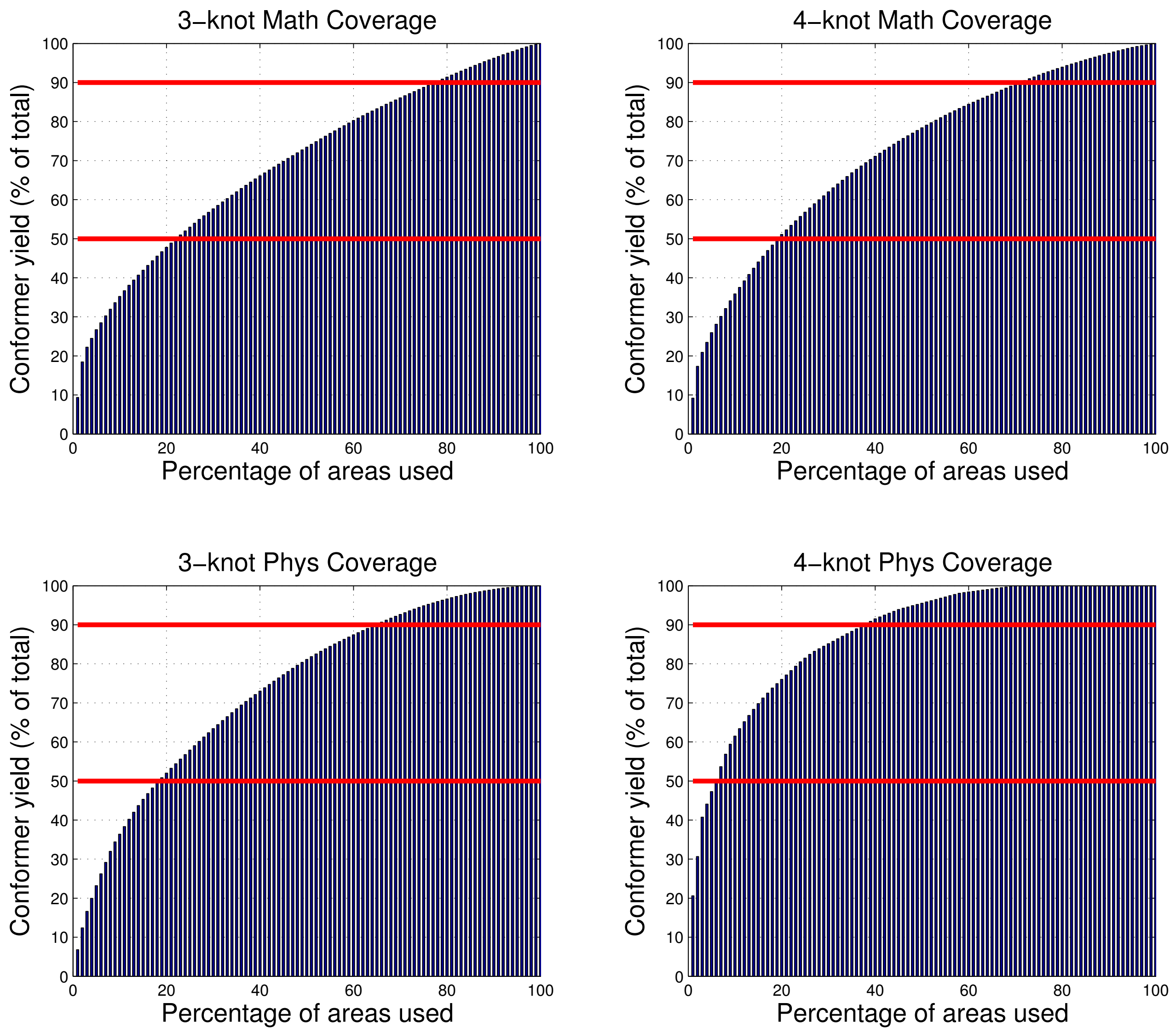

http://mc.manuscriptcentral.com/tandf/jenmol 